\title{
Amamentação e contato pele-a-pele no alívio da dor em recém-nascidos na vacina contra Hepatite B
}

\section{Breastfeeding and skin-to-skin contact for pain relief of newborns during hepatitis B vaccination}

Adriana Moraes Leite ${ }^{1}$, Ariadna de Cássia Tardim Oliveira da Silva ${ }^{2}$, Thaíla Corrêa Castral ${ }^{3}$, Lucila Castanheira Nascimento ${ }^{4}$, Mirna Isicawa de Sousa ${ }^{5}$, Carmen Gracinda Silvan Scochi ${ }^{6}$

\footnotetext{
${ }^{1}$ Enfermeira, Doutora em Enfermagem em Saúde Pública. Professora Doutor da Escola de Enfermagem de Ribeirão Preto (EERP) da Universidade de São Paulo (USP). Ribeirão Preto, SP, Brasil. E-mail: drileite@eerp.usp.br.

${ }^{2}$ Enfermeira, Mestre em Enfermagem em Saúde Pública. Enfermeira do Hospital Estadual de Ribeirão Preto. Ribeirão Preto, SP, Brasil. E-mail: silva.ariadna@yahoo.com.br

${ }^{3}$ Enfermeira, Doutora em Enfermagem em Saúde Pública. Professora Adjunto da Faculdade de Enfermagem da Universidade Federal de Goiás. Goiânia, GO, Brasil. E-mail: thaccastral@gmail.com.

${ }^{4}$ Enfermeira, Doutora em Enfermagem em Saúde Pública. Professora Associado da EERP/USP. Ribeirão Preto, SP, Brasil. E-mail: lucila@eerp.usp.br.

${ }^{5}$ Enfermeira. Discente do Programa de Pós-Graduação em Enfermagem, nível Doutorado, da EERP/USP. Enfermeira do Hospital das Clínicas da Faculdade de Medicina de Ribeirão Preto da USP. Ribeirão Preto, SP, Brasil. E-mail: miisicawa@gmail.com.

${ }^{6}$ Enfermeira, Doutora em Enfermagem. Professora Titular da EERP/USP. Ribeirão Preto, SP, Brasil. E-mail: cscochi@eerp.usp.br.
}

\section{RESUMO}

Tem-se como objetivo comparar a combinação entre o contato pele a pele com a amamentação, ao contato pele-a-pele durante a vacina contra a Hepatite B em recém-nascidos. Ensaio clínico randomizado cuja amostra constituiu-se de 55 $\mathrm{RN}$ a termo divididos entre os grupos contato pele-a-pele GP, $\mathrm{n}=38$ ), amamentação combinada ao contato pele-a-pele (GPA, n=27). A dor do RN foi avaliada por meio da mímica facial. Mensurou-se também a frequência cardíaca de todos os RN e frequência de sucção dos RN no GPA. Houve diferença estatisticamente significativa nos períodos de compressão e recuperação entre os grupos para os escores do NFCS. Os valores médios das FC foram significativamente mais elevados no GP na antissepsia/injeção. As sucções no GPA variaram de 10 a 28 sucções por minuto. A amamentação combinada ao contato materno pele-a-pele pode potencializar o efeito analgésico do contato materno pele-a-pele, contribuindo para uma melhor recuperação do RN após o procedimento.

Descritores: Recém-nascido; Dor Aguda; Aleitamento Materno; Vacinas; Enfermagem Neonatal.

\section{ABSTRACT}

The objective was to compare the combination of skin-to-skin contact with breastfeeding, to skin-to-skin contact during hepatitis B vaccination in newborns. Randomized clinical trial with a sample of 55 full-term newborns divided between two groups, namely; skin-to-skin contact $(G P, n=38)$, and breastfeeding combined with skin-to-skin contact (GPA, $n=$ 27). The pain of newborns was assessed by facial movements. The heart rate (HR) of all newborns was measured, as well as the suckling frequency of newborns in the GPA. There was a statistically significant difference in the periods of compression and recovery between the groups for the NFCS scores. The average values of HR were significantly higher in the GP during antisepsis/injection. The suckling in the GPA ranged between 10-28 suckles per minute. The breastfeeding combined with maternal skin-to-skin contact can enhance the analgesic effect of maternal skin-to-skin contact, contributing to a better recovery of newborns after the procedure.

Descriptors: Infant, Newborn; Acute Pain; Breast Feeding; Vaccines; Neonatal Nursing. 


\section{INTRODUÇÃO}

Durante as primeiras horas de vida os recém-nascidos (RN) sofrem várias alterações fisiológicas para se adaptarem a um ambiente aéreo ${ }^{(1)}$, além de depararemse com um ambiente iluminado, ruidoso, com odores diferentes e com perda do limite físico intrauterino ${ }^{(2)}$. Somando-se a esses fatores estão as rotinas pósnascimento, em que os $\mathrm{RN}$, em um curto período de tempo, são submetidos a procedimentos estressantes e, muitas vezes, dolorosos, dentre os quais a administração da vacina contra Hepatite $B^{(3)}$.

A vacinação constitui-se em uma estratégia eficaz para a prevenção de doenças infectocontagiosas e redução da mortalidade infantil, no entanto, a dor é um efeito adverso esperado ${ }^{(4)}$, e quando não tratada, pode acarretar em consequências negativas para a criança em procedimentos futuros, tais como, medo antecipado, hipersensibilidade a dor ${ }^{(5)}$, redução da efetividade dos analgésicos $^{(6)}$ e medo de agulhas ${ }^{(7)}$.

Diversas medidas são recomendadas para aliviar a dor durante a vacinação em RN, como a amamentação, soluções adocicadas, anestésicos tópicos e distração ${ }^{(8)}$. Também há evidência do efeito analgésico aditivo do contato pele-a-pele combinado à glicose $25 \%$ na redução do escore de dor durante a vacina contra a Hepatite B em RN a termo ${ }^{(9)}$. No entanto, com exceção deste último estudo, nenhum outro investigou medidas para reduzir a dor da injeção em RN durante a vacina da Hepatite B.

Considerando-se a frequente exposição dos RN a esse procedimento e a necessidade de um cuidado mais humanizado no processo de nascimento, é emergente a realização de estudos que envolvam a investigação da eficácia de medidas não farmacológicas para o alívio da dor na administração da vacina contra a Hepatite B.

Alguns estudos mostram evidências na eficácia da amamentação materna como medida não farmacológica para o alívio da dor, durante a administração de injeção intramuscular em crianças a partir de dois meses de idade e durante a administração da vacina tríplice bacteriana (DPT-tétano, difteria e coqueluche) ${ }^{(10-11)}$.
Um estudo verificou que a amamentação foi mais eficaz do que o spray analgésico local em bebês de até seis meses de idade durante a administração das vacinas contra a Hepatite B e DPT ${ }^{(12)}$.

Com relação a medidas não farmacológicas com participação da mãe para o alívio da dor aguda neonatal, pesquisa realizada com $\mathrm{RN}$ de até 24 horas de vida que foram amamentados dois minutos antes e durante a primeira dose comparados a recém-nascidos, que foram mantidos no colo materno apenas, concluiu que os recém-nascidos amamentados apresentaram menores escores de dor durante o procedimento ${ }^{(13)}$.

Outro estudo ao comparar três grupos: RN em contato pele a pele + amamentação, $\mathrm{RN}$ em contato pele a pele e RN mantidos no berço sem intervenção, não encontrou diferença estatística nos escores de dor entre o primeiro e segundo grupos, porém, o procedimento de dor avaliado foi a punção de calcâneo ${ }^{(14)}$.

Até onde se sabe, não há estudos que tivessem comparado a eficácia do contato pele a pele+ amamentação, comparado ao contato pele a pele durante a primeira administração da dose da vacina contra a Hepatite B. Assim, procurou-se no presente estudo comparar a combinação entre o contato pele a pele com a amamentação, ao contato pele-a-pele durante esse procedimento.

A hipótese da pesquisa é que a amamentação combinada ao contato pele-a-pele é mais efetiva do que o contato pele-a-pele na redução das manifestações comportamentais e fisiológicas de dor, durante a administração da injeção intramuscular para a vacina contra a Hepatite B.

Este estudo insere-se nas propostas do cuidado atraumático, desenvolvimental e humanizado ao $\mathrm{RN}$, buscando evidências científicas sobre intervenções maternas para o manejo da dor aguda decorrente da administração da vacina contra Hepatite B. Logo, o objetivo deste estudo foi avaliar o efeito da amamentação combinada ao contato pele-a-pele no 
alívio da dor dos RN submetidos à vacina intramuscular contra Hepatite B.

\section{MÉTODOS}

Ensaio clínico randomizado realizado numa maternidade do interior paulista, cujos critérios de seleção envolveram os $\mathrm{RN}$ a termo com até 12 horas de vida internados em alojamento conjunto, Apgar $\geq 7$ no 5 응 minuto de vida; em amamentação materna exclusiva sob livre demanda, que sugaram o seio materno ao menos uma vez após o nascimento, e cujas mães apresentavam condições físicas para amamentar. Foram excluídos os RN que foram amamentados até uma hora antes do procedimento.

A partir de estudos similares ${ }^{(2-15)}$ a amostra constituiu-se de $55 \mathrm{RN}$ a termo que foram divididos aleatoriamente por meio de um programa de randomização computacional em dois grupos: grupo contato pele-a-pele (GP, $n=38 \mathrm{RN}$ ), que foram mantidos por cinco minutos em berço comum (período basal), e logo após, posicionados em contato materno pele-a-pele durante 15 minutos (período de tratamento), seguido do período de antissepsia/injeção, e período de compressão com algodão embebido em álcool a $70 \%$ em contato pelea-pele, até cinco minutos após o término do procedimento (período de recuperação); e o grupo amamentação combinada ao contato pele-a-pele (GPA, $n=27$ ), que foram mantidos em berço comum por cinco minutos (período basal), e a seguir, foram posicionados em contato pele-a-pele durante cinco minutos, depois combinado à amamentação por mais 10 minutos, sendo mantidos nesta condição durante os períodos de antissepsia/injeção, compressão e a recuperação (cinco minutos após o término da compressão).

A dor do RN foi avaliada por meio da mímica facial e a frequência cardíaca ( $F C$ ) mensuradas nos períodos de tratamento, procedimento (antissepsia/injeção), compressão e recuperação.

A mímica facial foi mensurada por meio do Sistema de Codificação da Atividade Facial Neonatal (NFCS) ${ }^{(16-17)}$, validada no Brasil $^{(18)}$.Codificaram-se apenas as ações faciais fronte saliente, fenda palpebral e sulco nasolabial, pois a visualização de algumas ações da hemiface inferior estavam prejudicadas devido à amamentação ${ }^{(2)}$.

As faces dos RN foram filmadas com uma câmera SONY modelo DCR-DVD 650 e gravadas em uma mídia DVD por uma auxiliar de pesquisa. As imagens gravadas foram posteriormente codificadas e observadas por meio de um Televisor Sansung 29 polegadas.

As três ações faciais do NFCS foram codificadas a cada dois segundos durante os 20 segundos finais de cada período da coleta de dados. Para a obtenção dos escores do NFCS procedeu-se o seguinte cálculo para todos os períodos da coleta de dados: NFCS do período analisado (20 segundos) $=$ número de atividades observadas nos 10 intervalos de dois segundos / número máximo de atividades possíveis ou 30 (10 intervalos $X 3$ ações) $X 10^{(2)}$.

Para a mensuração da frequência cardíaca (FC) em todos os bebês foi instalado um monitor cardíaco POLAR - modelo RS 200, composto por uma unidade de pulso a qual registra e armazena os dados, e um transmissor codificado conectado a dois eletrodos de captação de frequência, localizados na região torácica do RN.

Os dados armazenados pela unidade de pulso foram transmitidos ao software polarweblink, responsável pela apresentação das frequências médias durante o tempo analisado. Foram analisados também número de situações de risco (FC<110 e >160 bat/min) ${ }^{(2)}$.

Também foi mensurada a frequência de sucção dos $\mathrm{RN}$ no GPA, desde o momento em que os RN iniciaram a mamada no período de tratamento (cinco minutos antes da administração da vacina) até o final do período de recuperação. Foi quantificada durante os diferentes períodos a cada 30 segundos inicialmente, até obter-se o número de sucções por minuto.

A injeção intramuscular para administrar a vacina contra a Hepatite $B$ foi realizada por apenas uma técnica de enfermagem treinada por meio de uma seringa $B D^{R}$ conectada a uma agulha Nipro $^{R}$ de calibre $20 \times 5,5$ no músculo vasto lateral da coxa direita. 
Os dados relacionados à paridade materna, nascimento (horas de vida do $\mathrm{RN}$, sexo, peso de nascimento, Apgar do 1ㅇ e 5o minuto), tempo decorrido desde a última mamada no momento da imunização do RN e número e tipo de procedimentos dolorosos recebidos pelos $\mathrm{RN}$ até o momento do procedimento foram obtidos por meio da revisão do prontuário médico.

O estudo foi aprovado pelo Comitê de Ética em Pesquisa da Escola de Enfermagem de Ribeirão Preto (protocolo 002/2008) e a coleta de dados foi iniciada após o consentimento das mães participantes.

Para avaliar a fidedignidade da codificação do NFCS foi realizado o teste de Coeficiente de Correlação Intra Classes (ICC), a partir da observação independente das filmagens da face dos $\mathrm{RN}$ feita pela pesquisadora e uma auxiliar de pesquisa. Para tal, foi utilizada uma amostra aleatória de $11 \mathrm{RN}$, sendo seis do GPA e cinco do GP. Um total de 20 valores de ICC foi obtido para cada uma das ações faciais (fronte saliente, fenda palpebral e sulco nasolabial). O valor médio de ICC obtido foi de 0,8801( $\pm 0,1434)$, mostrando excelente concordância entre os observadores.

O teste Mann Whitney foi utilizado para verificação das diferenças entre os escores de NFCS entre os grupos e períodos (intragrupos) e para a verificação de diferenças entre os escores de FC entre os grupos e períodos (intragrupos), ANOVA com medidas repetidas sendo que o nível de significância utilizado foi de 0,05 $(\alpha=0,05)$.

\section{RESULTADOS}

A Tabela 1 mostra os dados de nascimento do RN em ambos os grupos. Verifica-se que não houve diferença significativa entre os grupos com relação ao peso ao nascer, Apgar no um e cinco minutos, tempo de última mamada e horas de vida.

Tabela 1: Valores médios e desvios padrão dos dados de nascimento dos RN. Ribeirão Preto, SP, Brasil, 2010.

\begin{tabular}{|c|c|c|c|c|c|}
\hline \multirow{2}{*}{ Variáveis } & \multicolumn{2}{|c|}{ GP } & \multicolumn{2}{|c|}{ GPA } & \multirow[b]{2}{*}{$p$} \\
\hline & $\overline{\bar{x}}$ & $D P$ & $\bar{x}$ & $D P$ & \\
\hline Peso ao nascer (g) & 3170,71 & 351,912 & 3113,33 & 425,445 & $0,5871^{1} *$ \\
\hline Apgar $1^{\circ}$ minuto & 9,18 & 0,723 & 8,78 & 0,751 & $0,62^{2} *$ \\
\hline Apgar $5^{\circ}$ minuto & 9,82 & 0,476 & 9,89 & 0,476 & $0,6942^{2} *$ \\
\hline Tempo da última mamada $(\mathrm{h})$ & 2,14 & 0,932 & 1,85 & 0,718 & $0,2011^{1} *$ \\
\hline Horas de vida & 6,93 & 0,766 & 6,93 & 0,730 & $0,801^{2} *$ \\
\hline
\end{tabular}

${ }^{1}$ Teste $t$ de Student.

${ }^{2}$ Mann Whitney.

* Não houve diferença estatisticamente significante entre as médias dos grupos.

No GP havia 15 RN do sexo feminino $(53,6 \%)$ e 13 do sexo masculino $(46,4 \%)$; o GPA foi composto por $15 \mathrm{RN}$ $(55,5 \%)$ do sexo feminino e $12 \mathrm{RN}$ do sexo masculino (44,5\%). O teste qui-quadrado mostrou não haver diferença entre os grupos quanto ao sexo dos $\mathrm{RN}$ $(p=1,00)$.

Dos 28 bebês do GP, nove receberam mais de um procedimento doloroso, enquanto que dos $27 \mathrm{RN}$ do GPA, cinco foram expostos a mais de um procedimento doloroso no período que antecedeu a injeção da vacina contra a Hepatite B. Os tipos de procedimentos dolorosos realizados foram: coleta de sangue arterial para exames de hemograma, Proteína C Reativa (PCR) e hemocultura, e coleta de sangue capilar para teste de glicemia. Em média, os RN do GP foram expostos a 1,32 $( \pm 0,48)$ procedimentos dolorosos e os RN do GPA a $1,19( \pm 0,40)$ procedimentos dolorosos, porém não houve diferença estatística significativa quanto ao número de procedimentos dolorosos entre os grupos $(p=0,251)$.

Todos os RN foram expostos a pelo menos um procedimento doloroso após o nascimento, sendo este a administração intramuscular da vitamina K.

Na Tabela 2 encontra-se a duração de cada período do procedimento, sendo que não houve diferença entre os grupos. 
Tabela 2: Valores médios, medianas e desvios padrão dos tempos gastos em segundos nos períodos de antissepsia, injeção e compressão, durante a administração da vacina contra Hepatite B nos grupos. Ribeirão Preto, SP, Brasil, 2010.

\begin{tabular}{|c|c|c|c|c|c|c|c|}
\hline \multirow{2}{*}{ Variáveis } & \multicolumn{3}{|c|}{ GP } & \multicolumn{3}{|c|}{ GPA } & \multirow{2}{*}{$p$} \\
\hline & $x$ & $M d$ & $D P$ & $x$ & $M d$ & $D P$ & \\
\hline Período de Antissepsia & 8,42 & 9,01 & 1,97 & 9,95 & 9,59 & 5,26 & $0,200^{1 *}$ \\
\hline Período de injeção da vacina & 7,32 & 7,59 & 2,07 & 9,26 & 8,0 & 5,56 & $0,090^{1 *}$ \\
\hline Período de Compressão & 19,78 & 19,59 & 3,80 & 21,25 & 20,0 & 3,69 & $0,051^{1 *}$ \\
\hline
\end{tabular}

${ }^{1}$ teste t de Student.

* Não houve diferença estatisticamente significante entre os grupos.

Os valores do escore do NFCS foram menores no GPA em todos os períodos do procedimento, destacando-se que houve diferença estatisticamente significativa nos períodos de compressão e recuperação (Tabela 3).

Tabela 3: Comparação dos escores de NFCS entre recém-nascidos dos grupos GP e GPA. Ribeirão Preto, SP, Brasil, 2010.

\begin{tabular}{|c|c|c|c|c|c|}
\hline \multirow[t]{2}{*}{ Escore NFCS } & \multicolumn{2}{|c|}{ GP } & \multicolumn{2}{|c|}{ GPA } & \multirow{2}{*}{$p$} \\
\hline & $x$ & $D P$ & $x$ & $D P$ & \\
\hline Tratamento & 1,21 & 2,36 & 1,15 & 2,07 & $0,965^{2^{*}}$ \\
\hline Antisepsia/Injeção & 19,36 & 3,95 & 18,44 & 3,75 & $0,461^{2^{*}}$ \\
\hline Compressão & 11,86 & 5,45 & 6,44 & 4,05 & $0^{2 * *}$ \\
\hline Recuperação & 9,43 & 3,14 & 6,37 & 1,964 & $0^{2^{* *}}$ \\
\hline
\end{tabular}

${ }^{2}$ Mann Whitney.

* Não houve diferença estatisticamente significante entre os grupos.

** Houve diferença estatisticamente significante.

Quanto à FC, os valores médios foram de tratamento para a antissepsia/injeção, significativamente mais elevados no GP em relação ao GPA no período de antissepsia/injeção (Tabela 4). É importante destacar que na análise intragrupos, houve antissepsia/injeção para a compressão e antissepsia/injeção para a recuperação em ambos os grupos separadamente.

diferença estatisticamente significativa entre os períodos

Tabela 4: Média e respectivo desvio padrão da FC (bpm) dos RN do GP e GPA em cada período do procedimento. Ribeirão Preto, SP, Brasil, 2010.

\begin{tabular}{|c|c|c|c|c|c|}
\hline \multirow{2}{*}{ Períodos } & \multicolumn{2}{|c|}{ GP } & \multicolumn{2}{|c|}{ GPA } & \multirow{2}{*}{$p$} \\
\hline & $x$ & $D P$ & $x$ & $D P$ & \\
\hline Basal & 136,7 & 0,9 & 138,3 & 0,9 & $0,2173^{*}$ \\
\hline Tratamento & 137,3 & 0,6 & 137,7 & 0,6 & $0,5943^{*}$ \\
\hline antissepsia/injeção & 161,7 & 0,9 & 157,9 & 0,9 & $0,0043 * *$ \\
\hline Compressão & 156,9 & 1,0 & 158,9 & 1,0 & $0,1583^{*}$ \\
\hline Recuperação & 144,2 & 0,6 & 144,3 & 0,7 & $0,8683^{*}$ \\
\hline
\end{tabular}

${ }^{3}$ ANOVA com medidas repetidas.

* Não houve diferença estatisticamente significante entre os grupos.

** Houve diferença estatisticamente significante.

Considerando a FC em relação aos grupos, o teste ANOVA com medidas repetidas mostrou haver diferença estatisticamente significativa na interação entre os dois grupos durante o período de antissepsia/injeção ( $p=0,004)$, ou seja, o GP apresentou valores de FC maiores durante a antissepsia/injeção do que o GPA.

Ainda nesse grupo, os valores médios da FC no período de antissepsia/injeção encontravam-se acima de 160 bpm, considerados como situação de risco.
O número de sucções no GPA variou de 10 a 28 sucções por minuto no período de tratamento, de zero a sete sucções por minuto no período de antissepsia/injeção, zero a 10 sucções por minuto no período de compressão e de nove a 21 sucções por minuto no período de recuperação (Tabela 5). 
Tabela 5: Valores médios e respectivo desvio padrão da sucção dos RN para o GPA de acordo com os períodos. Ribeirão Preto, SP, Brasil, 2010.

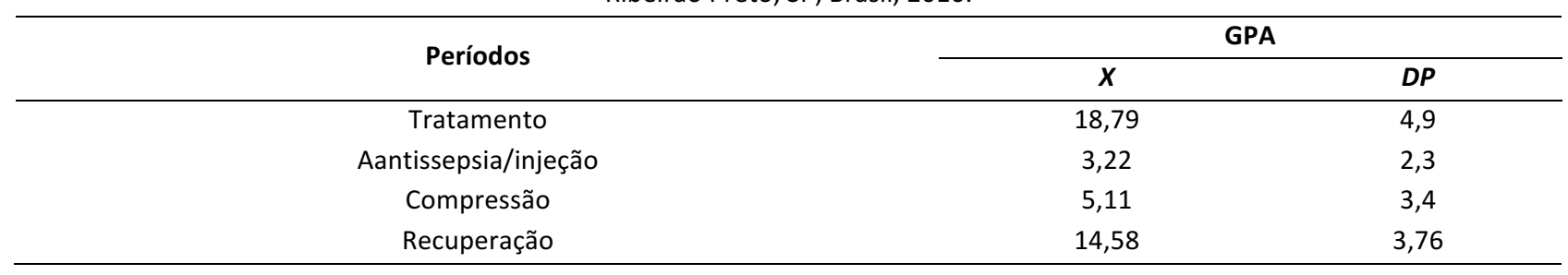

\section{DISCUSSÃO}

Ao comparar o efeito analgésico do contato pele-apele combinado à amamentação com o contato materno pele-a-pele no alívio da dor decorrente da injeção da vacina contra a Hepatite $B$, encontramos que os RN tratados com o contato pele-a-pele combinado à amamentação apresentaram menores escores do NFCS durante a compressão e recuperação do que aqueles que foram mantidos apenas em contato materno pele-a-pele. Dessa forma, os resultados do presente estudo apontam que a amamentação combinada ao contato materno pele-a-pele pode potencializar o efeito analgésico do contato materno pele-a-pele, contribuindo para uma melhor recuperação do $\mathrm{RN}$ após o procedimento da administração da vacina contra a Hepatite B.

Estudo anterior examinou o efeito combinado do contato materno pele-a-pele com outras intervenções não farmacológicas em RN a termo durante a punção de calcâneo $^{(19)}$. Em consonância com os nossos achados, o contato materno pele-a-pele combinado à amamentação ou à sacarose resultou em menor duração do choro em relação ao contato materno pele-a-pele sozinho, no entanto, os escores de dor foram menores no grupo que recebeu sacarose em relação aos demais grupos.

Não se sabe com convicção quais são os mecanismos analgésicos do contato materno pele-a-pele. Tal medida não farmacológica congrega diversos estímulos, tais como o cheiro, a voz, o calor, a textura da pele, o som dos batimentos cardíacos, o movimento do tórax durante a respiração materna, a contenção e o posicionamento em prona $^{(20)}$. Estudos com animais sugerem que os sistemas opióides e outros neuropeptídeos, como a colecistoquinina, estão envolvidos no aprendizado dos aspectos discriminativos maternos, assim como no estabelecimento do vínculo mãe e filho, fatores indispensáveis para a formação da autorregulação do $\mathrm{RN}^{(21)}$.

Ao se combinar o contato materno pele-a-pele com a amamentação, adicionou-se aos fatores acima descritos, as propriedades analgésicas oro-táteis da sucção e sensação gustativa e o efeito gástrico do leite materno ${ }^{(22)}$.É importante destacar que não se pode afirmar que todas as mães estavam com as mamas lactantes, uma vez que não se avaliou a presença de colostro nelas.

A frequência de sucções nos diferentes períodos foi semelhante a outra pesquisa realizada ${ }^{(2)}$, ou seja, os bebês mantiveram sucção efetiva no período de tratamento da dor, diminuindo na antissepsia/injeção, aumentando um pouco na compressão e voltando a sugar um pouco mais na recuperação. Note-se portanto que no momento da dor aguda, a frequência de sucção diminui bastante e depois deste momento os RN voltam a sugar procurando a recuperação. É importante destacar que a frequência de sucção foi uma variável que se destacou no presente estudo em comparação aos demais que utilizaram a amamentação na situação da administração da vacina contra a Hepatite $B$, pois não foi avaliada nesses estudos $^{(12-13)}$.

Quanto ao tempo de tratamento oferecido para o grupo dos RN amamentados, nos dois demais estudos encontrados na literatura, os mesmos foram amamentados dois minutos antes do procedimento ${ }^{(13)} \mathrm{e}$ imediatamente antes de iniciar o procedimento ${ }^{(12)}$, e em ambos continuando a mamar até o término. 0 presente estudo possui o diferencial, assim como em pesquisa 
anterior $^{(2)}$, de que os RN iniciaram a mamada cinco minutos antes da vacina, mantendo-se mamando durante o procedimento e cinco minutos na recuperação.

Um outro estudo implementou o contato materno pele-a-pele apenas dois minutos antes da injeção para vacina contra Hepatite $B$, tendo encontrado efeitos analgésicos favoráveis ${ }^{(9)}$.

Tanto a amamentação como o contato materno pelea-pele, constituem-se em estratégias já preconizadas pelo Ministério da Saúde ${ }^{(23)}$, sendo o alívio da dor aguda em RN mais um benefício que justifica a promoção do uso destas medidas durante a vacinação. Há uma particularidade a ser considerada em ambas as intervenções que é participação da mãe como o único agente desse cuidado. Com isso, o benefício para o $\mathrm{RN}$ se estende para a mãe, que também tem o seu estresse reduzido ${ }^{(24-25)}$.

\section{CONSIDERAÇÕES FINAIS}

Os resultados do presente estudo permitem recomendar o uso do contato materno pele-a-pele e da amamentação para a redução da dor decorrente da vacinação contra a Hepatite $\mathrm{B}$ em $\mathrm{RN}$ a termo, sendo a combinação destas intervenções com maior efeito analgésico na redução dos escores de dor durante a injeção e maior estabilidade fisiológica após a injeção do que as intervenções sozinhas.

A inclusão da avaliação do estado de sono e vigília e da duração do choro dos RN em ambos os grupos também poderia colaborar com os achados do estudo, uma vez

\section{REFERÊNCIAS}

1. Moore ER et al. Early skin-to-skin contact for mothers and their healthy newborn infants. Cochrane Database of Systematic Reviews 2012, Issue 5. Art. №.: CD003519. 2.Leite AM, Linhares MBM, Lander J, Castral TC, Santos CB, Scochi CGS. Effects of breastfeeding on pain relief in full-term newborns. The ClinicalJournalofPain. 2009; 25 (9): 827-32. 3. Ministério da Saúde. Atenção à saúde do recém-nascido. Guia para os profissionais de saúde. Cuidados Gerais.Brasília (Brasil): Ministério da Saúde, 2011. 195 p. que se tratam de variáveis consideradas complementares na avaliação da dor neonatal.

Considerando-se que não foi controlado se todas as mães estavam com as mamas lactantes, para estudos futuros recomenda-se que tal variável seja avaliada, possibilitando a análise dos fatores que envolvem a presença do leite como medida antinociceptiva em situação similar.

Outro aspecto que merece ser melhor avaliado em estudos futuros é o comportamento materno durante a amamentação e o contato materno pele-a-pele, visto que estas estratégias possibilitam a participação ativa da mãe e podem modificar a resposta do RN ao evento doloroso.

O tratamento da dor neonatal compreende uma das ações de grande relevância da equipe de saúde para o bem-estar do bebê, visto que a dor interfere no restabelecimento de sua saúde, podendo apresentar repercussões a longo prazo em relação à integração da criança na sua família, ao desenvolvimento cognitivo e à aprendizagem, refletindo-se, também, no aumento da morbimortalidade.

Para a implementação efetiva dessas medidas aqui recomendadas é necessário que os profissionais de saúde se conscientizem do direito dos $\mathrm{RN}$ de terem a dor evitada e tratada.

Espera-se que os resultados do presente estudo tragam subsídios para transformações na prática assistencial em Neonatologia, contribuindo com a melhoria da qualidade do cuidado e de vida das crianças e suas famílias.

4.Taddio A, Chambers CT, Halperin SA, Ipp, Locket D, Reider $M J$, Shah V. Inadequatepain Management duringroutinechildhoodimmunizations: theneverof it. ClinTher. 2009; 31(sup.B): S 152-67.

5. Taddio A, Ipp M, Kikuta A, Shah V. Physical interventions and injection techniques for reducing injection pain during routine childhood immunizations: systematic review of randomized controlled trials and quasi-randomized controlled trials. ClinTher. 2009; 31, (Suppl 2): S48-76

6. Steven BJ, Abbott LK, Yamada J, Harrinson D, Stinson J, Taddio LK, Babwick M, Latimer M, Scatt SD, Rashotte J, 
Campbell F, Finley GA. Epidemiology and managment for procedure pain in children in Canadian hospitals. Canadian Medical Association Journal 2011; 19 (183): E403-412.

7.Akuma $A O$, Jordan S. Pain management in neonates: a survey of nurses and doctors. Journal of Advanced Nursing. 2012; 68 (6): 1288-1301.

8.Taddio A, Appleton M, Bortolussi R, Chambers C, Dubey V, Halperin S, Hanrahan A, Ipp M, Lockett D, MacDonald N, Midmer D, Mousmanis P, Palda V, Pielak K, Riddell RP, Rieder $M$, Scott J, Shah V.Reducing the pain of childhood vaccination: an evidence-based clinical practice guideline. CMAJ. 2010; 182(18): 843-455.

9.Chermont AG, Falcão LFM, Silva EHLS, Balda RCX; Guinsburg R. Skin-to-Skin Contact and/or Oral 25\% Dextrose for Procedural Pain Relief for Term Newborn Infants. Pediatrics. 2009; 124(6):1101-1107.

10.Efe E, Ozer Z. The use of breastfeeding for pain relief during neonatal immunization injection. Applied Nursing Research. 2007;20 (1) 10-16.

11. SHAH, PS. Breastfeeding or breast milk for procedural pain in neonates (Review). The Cochrane Library; 2012 Issue 12. 12.Khadijeh B, Khodaei F, Zahra A, Marouti MJ. Nurs Midwifery. 2013; 18 (91): 3-37.

13. Modarres M, Jazayeri A, Rahnama P, Montarezi A. Breastfeeding and pain relief in full-term neonates during immunization injections: a clinical randomized trial. Anesthesiology. 2013; 13(22): 1-6.

14.Okan F, Ozdil A, Bulbul A, Yapici Z, Nuhoglu A. Analgesic effects of skin-to-skin contact and breastfeeding in procedural pain in healthy term neonates. Ann Trop Paediatr. 2010; 30(29): 118-28.

15.Castral, TC.; Warnock, F.; Leite, AM.; Haas, VJ;.Scochi, CGS. The effects of skin-to-skin contact during acute pain in preterm newborns. European Journal of Pain. 2008; 12(4): 464-71. 16.Grunau, RV.; Craig, KD. Neonatal Facial Coding System Revised: training manual. Vancouver: Early Human Experience Unit; 2010. 18 p.

17.Guinsburg R, Arias MCC. A Linguagem da dor no recémnascido. São Paulo: Sociedade Brasileira de Pediatria; 2010. 18. Guinsburg R, Balda RCX, Berenguel RC, Almeida MFB, Tonelotto J, Santos AM, Kopelman BI.

Behavioralpainscalesassessment in neonates. J Pediatr. 1997; 73(6): 411-8.

19.Marín GMÁ, Del Rey HB, Jiménez FL, Medina V, Iglesias FB, Vázquez RM, Escudero HV, et al. Analgesia with breastfeeding in addition to skin-to-skin contact during heel prick. Arch Dis Fetal Neonatal Ed. 2013; 98 (6):499-503.

20.Nimbalkar SM, Chaudhary NS, Gadhavi KV, Phatak A. Kangaroo Mother Care in Reducing Pain in Preterm Neonates on Heel Prick. Indian J Pediatr. 2013; 80(1): 6-10.

21. Weller A, Feldman R. Emotion regulation and touch in infants: the role of cholecystokinin and opioids. Peptides. 2003; 24(5):779-88.

22.Castral TC, Leite, A. M.; Gilbert M J, Mendes T R, Hisamitsu L $B$, Scochi CGS. Intervenções maternas para o alívio da dor aguda no recém-nascido. RevistaQualidade do Hospital das Clínicas. 2009; 1:23-27.
23.Ministério da Saúde. Normas de atenção humanizada ao recém-nascido de baixo peso: método canguru, Brasília (DF): Secretaria de Políticas de Saúde. Área Técnica de Saúde da Criança. Brasília: Ministério da Saúde; 2009. 238p.

24.Castral TC, Warnock FF, Ribeiro LM, de Vasconcelos MG, Leite AM, Scochi CG. Maternal factors regulating preterm infants' responses to pain and stress while in maternal kangaroo care. Rev Lat Am Enfermagem. 2012. 20(3):435-43. 25. Warnock, F.; Castral, T.C.; Brant, R.; Sekilian, M.; Leite, A.M.; Owens, S.P.; Scochi, C.G.S. Brief Report: Maternal Kangaroo Care for Neonatal Pain Relief: A systematic Narrative Review. Journal of Pediatric Psycology. 2010; 35(9):975-84.

Recebido: 17/09/2014.

Aceito: 02/06/2015.

Publicado: 30/09/2015. 